\title{
Menopausal Arthritis- A Lesser-Known Problem -A Case Study
}

ISSN: 2576-8875

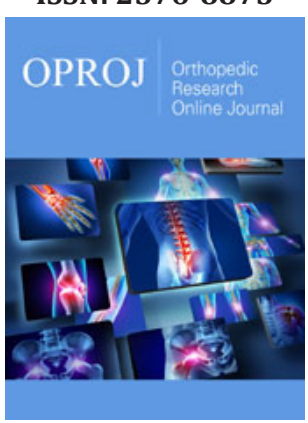

*Corresponding author: Sindhu Madiwalar, Karnataka State Rural Development and Panchayat Raj University, India

Submission: 侮June 29, 2021

Published: 恝July 08, 2021

Volume 8 - Issue 3

How to cite this article: Sindhu Madiwalar, Suresh Kishanrao. Menopausal Arthritis- A Lesser-Known Problem -A Case Study. Ortho Res Online J. 8(3). OPROJ. 000688. 2021.

DOI: 10.31031/OPROJ.2021.08.000688

Copyright@: Sindhu Madiwalar, This article is distributed under the terms of the Creative Commons Attribution 4.0 International License, which permits unrestricted use and redistribution provided that the original author and source are credited.

\author{
Sindhu Madiwalar ${ }^{1 *}$ and Suresh Kishanrao ${ }^{2}$ \\ ${ }^{1}$ B.Sc., (MPH), Karnataka State Rural Development and Panchayat Raj University, India \\ ${ }^{2}$ Public Health Consultant \& Visiting Professor-MPH, India
}

\begin{abstract}
Menopausal arthritis, a form of osteoarthritis manifests as joint pain and inflammation, that occurs early in menopause and may continue to get worse as time goes on. It is well known that Estrogen helps to reduce inflammation in women and there exists a relationship between falling estrogen levels and arthritis though the exact mechanism is still not fully clear. During menopause when estrogen levels decline, Hot flashes, Night sweats, Mood swings, Fatigue, Weight gain and Vaginal dryness are common symptoms experienced. One of the lesser-known result is increase in inflammation, causing discomfort pain, swelling and stiffness of the joints and the condition is called 'menopausal arthritis'. Early menopause has higher risk of all associated health problems. Menopause itself should set in around the age of 4647 years in Indian women. We present a case of early menopause onset (as indicated by first delayed and irregular menses) accompanied by joint pains not responding to routine analgesics. A 44-year-old, housewife, reported with swelling and pain in both the ankles, toes and foot pain in November 2020, to a local practitioner. After physical examination and some basic investigations, he treated her with analgesics and anti- inflammatory drugs but with poor results in the remission of the pain. During the next menstrual cycle, which was delayed by 2 weeks (after 6 weeks instead of 4 weeks she was used to) the number of joints involved, and severity of the pain increased. This time they consulted a private Orthopedic specialist in the nearest town, who again after a battery of investigations including serological assessment of Rheumatoid Arthritis factor made provisional diagnosis of 'Polyarthritis with seronegative RA- Menopausal Arthritis. She was put on a combination of corticosteroids, NSAIDs and anti-rheumatoid drugs since mid- May 2021. While the follow-up visit is due next week, she has nearly $50 \%$ relief by now.
\end{abstract}

\section{Introduction}

When a woman reaches menopause, her body undergoes drastic hormonal changes, most notable being reduction in estrogen levels, a primary female sex hormone. Most people associate menopause with hot flashes and mood swings. One of the lesser-known problems that menopausal women may have to deal with is arthritis. It is unclear exactly how falling estrogen levels impact the joints during and after menopause, but the relationship seems solid. Research has found that estrogen can impact every aspect of joint health including the bones, muscles, cartilage, and ligaments. Menopausal arthritis, a form of osteoarthritis manifests as joint pain and inflammation, occurs early in menopause and may continue to get worse as time goes on even after their other menopausal symptoms have disappeared [1].

Menopause is a natural part of the aging process. A woman is considered in menopause when she has gone 12 months without menstruating. The average age at menopause in India, is 46.6 years. The first stage of menopause - perimenopause that begins during the early to mid-40s and can last from three to five years. Average age of menopause of an Indian woman is much less than their Western counter parts (51 years). A definite rural and urban division is also seen. A correlation between the age of menopause and social and economic status, married status, and parity status has been observed. BMI is the major determinant of 
endogenous estrogen levels. Women with higher BMI are supposed to have higher levels of estradiol (E1) and estrogen (E2) in their body, leading to a delayed menopause [2].

Fortunately, many of menopausal diseases can be prevented by timely intervention of lifestyle modification, menopausal hormone therapy, or mineral and vitamin supplementations such as calcium, Vitamin D, and micronutrients [1]. Since the fertility potential of Indian women starts compromising early, so we need to start with the preventive measures much early.

\section{Case Presentation}

A young woman of 44-year-old, a housewife, reported with swelling and pain in both the ankles, toes and foot pain in November 2020. She visited a local practitioner. After physical examination and some basic investigations, he treated her with analgesics and anti- inflammatory drugs but with poor results in the remission of the pain.

In December 2021, during her subsequent delayed menstrual periods (after 6 weeks instead of regular 4 weeks), she suffered the swelling, stiffness, and pain in more number of joints, like ankles, knees, shoulders and back and severity of the pain was also much more, therefore they consulted a private Orthopedic specialist in the nearest town.

\section{Physical examination}

On physical examination he noted swollen and tender ankles, shoulders, and knees, that were warm to touch. There was stiffness of muscles surrounding the affected joint and reduced range of movements. Her Pulse-70/min, BP-90/160, temperature- $98.2^{\circ} \mathrm{F}$ and SPO2- 99\% and all other parameters were normal.

\section{Investigations}

He got done a battery of tests like, Blood sugar, Cholesterol, LFT, Thyroid hormones, and $\mathrm{Hb} \%$ and serological RA factor, all of them were within normal limits.

\section{Diagnosis}

Based on the normal test results, ruling out all-possible causes of arthritis and negative RA tests he made a provisional diagnosis of 'Polyarthritis with seronegative RA- Menopausal Arthritis.

\section{Treatment}

He started symptomatic treatment.

1. Tablet Orthocort $6 \mathrm{mg}$. one early in the morning.

2. Lefno-20 (Leflunomide, drug used for active Rheumatoid arthritis) one tablet after meals in the afternoon,

3. Tablet Etova-ER 400 \{ETODOLAC (Non-Steroidal AntiInflammatory Drugs- NSAIDs\} after dinner and Bonflex Gel (is a massage gel with immediate warming effect formulated with natural ingredients which, when applied before and after any physical effort improves pain caused by bumps or tears in joints and tendons. It is perfect for preventing inflammation and preventing tendons and bone wear and tear) to be applied locally in the night before going to bed.

\section{Advises}

She has been advised consume more of raw turmeric and ginger (anti-inflammatory spices), fresh fruit and vegetables, whole grains, and protein, and minimize sugars and Ghee.

\section{Past menstrual history}

The menarche had started at the age of 13 years, had normal menstrual cycles after 2 years. She has 3 children all girls. The onset of Menopause was early by the Indian standards at the age of 44, with periods becoming irregular 45-50 days. There is no history of her mothers or sibling suffering from similar Menopausal arthritis or Rheumatoid arthritis.

\section{Discussions}

Perimenopause and menopause are triggered by hormones that fluctuate and decrease. Many parts of the body are affected as hormonal levels decline. These include- reproductive system, vaginal tract, urinary system, nervous system, heart, brain, bones, skin. Both pre-Menopause and menopause may cause joint pain, though it is much less than other disturbances listed. It affects the knees, shoulders, neck, elbows, or hands as was observed in our case study. Old joint injuries may begin to ache. One may start to notice that one felt more aches and pains in those bones, joints and muscles than she used to. Estrogen helps to reduce inflammation, but when menopause sets in its levels decline, that may increase inflammation, causing menopause-related arthritis. In the case under study, we observed the joint pains from the first irregular menstrual cycles. The diagnosis is not easy for a general practitioner as many of them do not know or even think of such a possibility as it happened in our case. An Orthopedic specialist or an obstetrician suspects and goes of Rheumatoid serological test and either confirms that as Rheumatoid arthritis or Poly arthritis with seronegative RA or Menopausal arthritis as did the Orthopedic in our case [3-7].

\section{Diagnosis}

Though Signs and symptoms of menopause, associated with joint pains are usually enough to talk about onset of the menopausal arthritis, specialist like to rule out other causes of arthritis. They may ask for testing.

A. Follicle-stimulating hormone (FSH) and estrogen levels. FSH levels increase, and estradiol levels decrease as menopause occurs [8].

B. Thyroid-stimulating hormone (TSH) because hypothyroidism can also cause symptoms like those of menopause.

C. In India most used test is Serological Anti-cyclic citrullinated peptide Test: This test looks for a specific autoantibody called anti-cyclic Citrullinated Peptide (anti-CCP). It is present in around $75 \%$ of people with Rheumatoid Arthritis 
(RA). Persons with more than 20 units of CCPs are considered as having RA factor positive. If a person has RA and anti-CCP antibodies, they have seropositive RA. People with seronegative RA have RA, but do not have anti-CCP antibodies. High levels of anti-CCP are present in around $97 \%$ of people with RA. The interpretation table is as below:

\begin{tabular}{|c|c|}
\hline Results & Meaning \\
\hline $\begin{array}{c}\text { Positive CCP antibodies and } \\
\text { positive RF }\end{array}$ & The person has RA. \\
\hline $\begin{array}{c}\text { Positive CCP antibodies and } \\
\text { negative RF }\end{array}$ & $\begin{array}{c}\text { The person has early-stage } \\
\text { RA/ will likely develop it. }\end{array}$ \\
\hline $\begin{array}{c}\text { Negative CCP antibodies and } \\
\text { negative RF }\end{array}$ & $\begin{array}{c}\text { There is a lower chance of } \\
\text { having RA. }\end{array}$ \\
\hline
\end{tabular}

The standard approach to relieve joint pain are: (1) Lifestyle changes, (2) Alternative medicine, and (3) Conventional medicine, in order of preference [7].

Lifestyle changes: A woman's diet help improve overall health by preventing chronic conditions, reducing inflammation in the body, and helping maintain a normal weight. Ideally a menopause diet should include protein, healthy fats, and complex carbs that are rich in the nutrients like Phytoestrogens - plant-based compounds that act as weak estrogens in the body, helping to balance hormones and alleviate unpleasant symptoms like joint pain like Soy, oats, mung beans, alfalfa. Kale, tomatoes, Swiss chard, parsley, kiwi that have ample Vitamin $\mathrm{K}$ play an important role in bone and cartilage mineralization, that will aid in joint pain treatment. Walnuts, flax, fish, olive oil, Sacha inch containing Omega-3 fatty acids have been shown beneficial for reducing joint inflammation and pain and improve weight bearing capacity for women with arthritis. Eggs, mushroom, tuna, salmon, carrots preference as they contain Vitamin D can combat Vit D deficiency, among older adults and reduce pain in the joints and muscles of the upper or lower extremities and hips.

Regular exercise: Exercise is an important strategy in joint pain treatment. It helps support the joints, improve mobility, and range of motion, and also prevent weight gain. Healthy adult women are recommended to get at least 150 minutes of moderate-level exercises or 75 minutes of vigorous workouts per week. Women should focus on low-impact aerobics, such as walking, with gentle muscle-strengthening exercises, like stretches, yoga, or Pilates. Women with joint pain passing through menopause should avoid injury-prone activities.

Medications: The options for medications for joint pain focus on reducing inflammation and relieving pain.

a) Pain relievers, such as acetaminophen or ibuprofen, taken orally or through intramuscular injections relieve acute pain.

b) Corticosteroid injections can be given in the affected joint or orally to reduce inflammation, thus alleviating pain and swelling. c) Hormone replacement therapy (HRT) is effective but increases the risk of serious side effects and health risks, therefore be reserved for women with severe symptoms only.

The standards treatment recommended is use of NSAIDs, anti-inflammatory drugs, but we feel our Orthopedic treated overenthusiastically by giving anti-inflammatory, anti- rheumatoid and corticosteroid drugs simultaneously to show quick relief. Many studies show that postmenopausal women in India suffer from various vasomotor, physical as well as psychiatric problems related to menopausal hormonal changes with varied frequencies. Vasomotor symptoms are the leading problems in 34.5\% and 39.5\% rural and urban women, respectively. Genitourinary complaints, like

i) stress incontinence was in the range of $10.5 \%$ and $8.5 \%$ respectively in rural and urban communities followed by increased.

ii) urinary frequency $9 \%$ and $7.5 \%$ respectively in rural and urban areas.

iii) Vaginal dryness was reported by $2.5 \%$ of rural women and $6 \%$ of urban women. Vaginal discharge was reported by $7 \%$ of rural and $4 \%$ of urban women. Uterine Prolapse was reported by $6 \%$ of rural women and $3.5 \%$ of urban women [3].

A community-based cross-sectional study carried out in an urban resettlement colony in South Delhi indicated the prevalence of $47 \%$ of knee osteoarthritis in women aged $\geq 40$ years. Osteoarthritis was diagnosed by using clinical criteria. Prevalence of osteoarthritis was found to be increased with age [4]. Another community-based cross-sectional study was conducted between January 1, 2016, and December 31, 2016, as per the menopausespecific quality of life questionnaire. A total of 345 postmenopausal women aged 40-60 years residing in the field practice area of Urban Health Centre, Ashok Nagar, Belagavi, Karnataka were interviewed at homes [5]. This study reported women complaining of weakness (75.7\%), ache in joints and muscles (72.8\%), low stamina (65.8\%), low backache (64.1\%), Sleep disturbances (54.5\%), involuntary Flatulence $(49.6 \%)$, urination on laughing or coughing $(39.7 \%)$, weight gain (39.4\%), frequent urination (38.6\%), changes in texture (dry) of skin (38.6\%), increased facial hair growth $(25.5 \%)$ compared to pre-menopause times [5].

A PAN- India survey from 21 cities involving 2108 individuals \{(including 401 perimenopausal women and 1707 postmenopausal women), (1415 had natural menopause and 292 had surgical menopause post-hysterectomy)\} was done in 2016. The study revealed that Age of menopause is a particularly important biomarker of an increased risk for various mid-life diseases and problems like obesity, hypertension, mental disorders. While a positive correlation was reported between marital status, duration of the marriage, socio-economic status, BMI, and education, a negative correlation was noted with parity and age at natural menopause. The onset of menopause did not exhibit any significant 
variation by age at menarche, although the association was positive [2].

A systematic review of 202 studies in India revealed the average age at menopause in India, is 46.6 years, while it ranged from a minimum of 44.69 years to a maximum of 48.95 years. Our case falls in the minimum category of this study. The age at menopause did not exhibit any significant variation by age at menarche, although the association was positive [6]. A Chinese study has shown that Follicle-stimulating hormone (FSH) and luteinizing hormone ( $\mathrm{LH}$ ) play an important role in bone mass regulation in postmenopausal women.

\section{Conclusion}

Many studies show that postmenopausal women in India suffer from various vasomotor, physical as well as psychiatric problems related to menopausal hormonal changes, but Menopausal arthritis needing treatment is less known.

1. General practitioners usually miss though signs and symptoms of menopause, associated with joint pains are usually enough to suspect.

2. Orthopedic and Gynecology specialist like to rule out other causes of arthritis especially rheumatoid arthritis by getting investigations like Serological RA factor, hypothyroidism, anemia, blood sugar, Cholesterol etc. and other causes of joint pains.

3. Management uses three approaches of diet, exercises and use of conventional medicines, like NSAIDs, Corticosteroids in the form of injections or oral tablets based on the severity of the pain and inflammation and local application on affected joints.

\section{Take home messages}

a) Menopausal arthritis is less known occurs early in menopause and may continue to get worse as time goes on as joint pain and inflammation. b) Diagnosis needs to rule out other causes of arthritis and treatment may last long.

c) Diet containing Phytoestrogens, Vitamins K \&D, Omega 3 fatty acids, exercises - 30 minutes a day and drugs NSAIDs and Corticosteroids are the main stay of management.

\section{References}

1. Coping-with menopausal-arthritis.

2. Maninder Ahuja (2016) Age of menopause and determinants of menopause age: A PAN India survey by IMS. J Midlife Health 7(3): 126131.

3. Ankita G, Neha M, Shraddha D (2017) A comparative study of morbidity among postmenopausal women of Rural \& Urban Allahabad, Uttar Pradesh, India. International Journal of Research in Medical Sciences $5(2)$.

4. Harsh Salve, Vivek G, Palanivel C, Kapil Y, Bir S (2010) Prevalence of knee osteoarthritis amongst perimenopausal women in an urban resettlement colony in South Delhi. Indian J Public Health 54(3): 155157.

5. Nidhi Pathak, Shivaswamy MS (2018) Prevalence of menopausal symptoms among postmenopausal women of urban Belagavi, Karnataka. Indian Journal of Health Sciences and Biomedical Research Kleu 11(1): 77-80.

6. Prasad JB, Naresh T, Pradyuman V (2021) Age at menopause in India: A systematic review. Diabetes Metab Syndr 15(1): 373-377.

7. (2021) Joint-Pain Treatments. Menopausal Transition.

8. Zhang Xu, Ai Hong, Xian Wu, Hong Z, Zhi Feng, et al. (2009) Relationship of age-related concentrations of serum FSH and LH with prevalence of osteoporosis in native Chinese women 400(1-2): 8-13. 